\title{
Expression of neutrophil gelatinase-associated lipocalin in colorectal neoplastic progression: a marker of malignant potential?
}

\author{
M H McLean ${ }^{*, 1}$, A J Thomson ${ }^{1}$, G I Murray ${ }^{2}$, N Fyfe ${ }^{2}$, G L Hold ${ }^{1}$ and E M El-Omar ${ }^{1}$ \\ ${ }^{1}$ Gastrointestinal Research Group, Division of Applied Medicine, University of Aberdeen, Foresterhill, Aberdeen AB25 2ZD, UK \\ and ${ }^{2}$ Department of Pathology, School of Medicine and Dentistry, University of Aberdeen, Foresterhill, Aberdeen AB25 2ZD, UK
}

Background: Neutrophil gelatinase-associated lipocalin (NGAL) has a diverse functional repertoire, involved in the innate immune response as well as cell growth and differentiation. Expression has been linked to malignant disease development and progression.

Methods: Neutrophil gelatinase-associated lipocalin expression was assessed immunohistochemically in 98 colorectal neoplastic lesions (52 cancer polyps (CaPs) and 46 sporadic adenoma/adjacent normal mucosa paired specimens) to investigate association with adenoma progression and early colorectal carcinogenesis.

Results: Within CaPs, all adenomatous and carcinomatous epithelium expressed NGAL, with 92\% (43 out of 47) and 58\% (19 out of 33) epithelial positivity, respectively, as well as positive stromal cell expression. This was significantly increased compared with normal mucosal epithelium $(P=0.0001)$. Neutrophil gelatinase-associated lipocalin positivity was also identified in sporadic lowgrade adenomas, in both the epithelial and stromal compartments as compared with adjacent normal mucosa $(P=0.0001$ and 0.0002), and this increased along with adenoma size $>1 \mathrm{~cm}(P=0.03)$.

Conclusion: Neutrophil gelatinase-associated lipocalin is expressed by the majority of human neoplastic colorectal lesions. This phenotypic switch occurs at an early stage in neoplastic progression with clear differential expression between normal mucosa and adenomatous polyps, rather than further downstream in disease progression at the adenoma-carcinoma transformation. Thus, NGAL expression is not a useful biomarker for determining disease progression from adenomatous to malignant colorectal neoplasia.

Neutrophil gelatinase-associated lipocalin (NGAL), also known as lipocalin-2 (LCN-2), is a member of a large lipoprotein superfamily with over 50 members involved in transmembrane transportation of small hydrophilic molecules (Bratt, 2000). Specifically, NGAL promotes the cellular internalisation of siderophores, and this underpins its role in the innate immune response; it mediates antibacterial action by depleting an essential microbial growth factor, iron particles, from the surrounding microenvironment. Interest in NGAL has grown over the last few decades, and it is now clear that this protein is linked to a spectrum of functions such as cell growth and differentiation, is systemically released as a pro-inflammatory signal and as such acts as a biomarker denoting risk and severity in a number of inflammatory conditions such as acute kidney injury, and can also be expressed from many cell types as a stress response. The biology of NGAL in health and disease has been elegantly reviewed recently by Chakraborty et al (2012). There is now a growing body of evidence revealing its potential role in development and progression of malignant disease, and indeed, increased NGAL expression is linked to cancer in a diverse array of body sites, such as breast, urinary tract, gynaecological, thyroid and gastric malignancies (Bolignano et al, 2010; Chakraborty et al, 2012).

Colorectal cancer is the second cause of cancer death in the Western world and accounts for significant morbidity and 
mortality (Haggar and Boushey, 2009). The framework of sporadic colorectal cancer is the adenoma-carcinoma sequence, describing the change from histologically normal cells to pre-malignant adenomatous epithelium exhibiting increasing degrees of cell dysplasia to overt invasive malignant disease (Fearon and Vogelstein, 1990; Leslie et al, 2002). The pre-malignant phenotype, colorectal adenomatous polyps, exerts characteristics that denote high-malignant potential, such as increasing size, increasing degree of cell dysplasia and greater villous content (O'Brian et al, 1990; Colucci et al, 2003; Giuliani et al, 2006). These features are central to the current guidelines on patient management and specifically interval of surveillance colonoscopy (Cairns et al, 2010). As the demand for colonoscopy increases, including those participating in the colorectal screening program, the identification of additional characteristics of high-malignant potential, such as the expression of a novel protein biomarker, may assist in risk stratification and judicious use of endoscopic resource.

There are a few reports linking NGAL expression to colorectal malignancy (Sun et al, 2011; Barresi et al, 2010, 2011). The most extensive of these from Sun et al (2011) assessed the expression of NGAL in a large number of tissue specimens from each stage of the adenoma-carcinoma sequence, and reported that this was indeed a marker of neoplastic progression. With specific reference to overt colorectal cancer, NGAL was significantly linked to increasing stage of disease, tumour recurrence and overall poorer prognosis. In addition, there have been reports of NGAL expression in early colorectal tumours, TNM stage 1, with a correlation to later survival (Barresi et al, 2010, 2011). With regard to neoplastic progression along the adenoma-carcinoma sequence, 'cancer polyps' (CaPs) offer an ideal and unique opportunity to investigate biological events surrounding the earliest point of carcinogenesis. These lesions by definition include adenomatous background histology with supervening carcinomatous change, evident by stromal reaction to invasive disease. Often these lesions also include histologically normal mucosa at the base, offering the opportunity to assess expression of a protein marker with each stage of disease development along the adenoma-carcinoma sequence, within a single lesion.

The aim of this study was to assess the expression of NGAL in the progression of colorectal neoplasia, first in a cohort of CaPs, then in a separate cohort of sporadic colorectal adenomas compared with adjacent normal colonic mucosa, in order to identify the point in the adenoma-carcinoma sequence where NGAL expression becomes prominent and hence assess whether this could be a biomarker of high-malignant potential in premalignant colorectal polyps.

\section{MATERIALS AND METHODS}

Tissue specimens. Neutrophil gelatinase-associated lipocalin expression was assessed in 98 colorectal neoplastic lesions in total. The initial cohort included 52 archival paraffin-embedded CaPs from 52 patients, identified from the pathology department diagnostic database, Aberdeen Royal Infirmary. All specimens were reviewed for confirmed histological evidence of normal colonic mucosa, adenomatous epithelium and an area of supervening adenocarcinoma by an expert gastrointestinal pathologist (GIM). The study was then expanded to include a further 46 sporadic colorectal adenomas and 26 adjacent normal colonic mucosal samples from 30 patients, collected from the Grampian colorectal cancer screening program, Aberdeen Royal Infirmary (McLean et al, 2011). Again, histological diagnosis was confirmed by an expert GIM. None of these patients had previously documented colorectal polyps, colorectal malignancy or a history of inflammatory bowel disease. In addition, there was no known heritable genetic abnormality documented, specifically none were noted to belong to a family with Familial Adenomatous Polyposis or Hereditary Non-polyposis colorectal cancer syndromes. Thus, this second sample cohort represents sporadic colorectal adenomas arising in a screened population.

Sections of $4 \mu \mathrm{m}$ were cut and placed onto 3-aminopropyltriethoxysilane-coated slides for subsequent immunohistochemical analysis. The macroscopic size of each polyp was measured formally as part of the pathological analysis post fixation and before embedding. This recorded size was used for all polyp specimens and therefore provided a consistent and precise measurement of size for all polyps included within this study.

Ethical approval was obtained from Grampian Ethics Committee.

Neutrophil gelatinase-associated lipocalin immunohistochemistry. Neutrophil gelatinase-associated lipocalin expression was determined immunohistochemically with polyclonal rabbit anti-human LCN-2 antibody (Sigma Aldrich, Dorset, UK: HPA002695; validated by Human Protein Atlas; www.proteinatlas.org) at a dilution of $1: 800$. Slides were dewaxed, rehydrated and subjected to antigen retrieval by microwaving for $20 \mathrm{~min}$ in $10 \mathrm{~mm}$ citrate buffer, $\mathrm{pH}$ 6.0. Tissue was stained using the Envision + peroxidase-linked biotin-free system on the DakoCytomation (Cambridgeshire, UK) Techmate Autostainer (Duncan et al, 2008; McLean et al, 2008, 2011). A 60min incubation with the primary antibody was incorporated within the staining protocol, and colour developed with diaminobenzidine. Sections were counterstained with haematoxylin. Tonsil was used as a positive control.

Evaluation of immunostaining. All stained sections were assessed microscopically by two observers (MHM and AJT) independently and noted to be positive or negative for NGAL expression. Localisation of positive immunoreactivity to either stromal cells, epithelial cells or both was documented at $\times 20$ magnification with the whole of the specimen scored over multiple HPFs. Within the $\mathrm{CaPs}$, this was performed for each histological compartment, i.e., normal mucosa, adenomatous epithelium and carcinomatous epithelium. The end score for each tissue type for both proportion of stained epithelial cells and intensity of staining was an average of those obtained from the total tissue assessment. If there was disagreement on staining pattern, the two observers assessed the case with a third reviewer (GIM) jointly, and any remaining issues were resolved by discussion.

The proportion of positive epithelial cell staining and intensity of epithelial cell staining were scored on a modified scaled system. For proportion of positive epithelial cells, the scale was as follows: $1 \leqslant 50 \%$ of total cell population, and $2 \geqslant 50 \%$ of total cell population. For positive immunoreactivity intensity, $1=$ weak/ moderate and $2=$ strong.

Statistical analysis. The association of NGAL expression with histological cell type and adenoma size was evaluated using the $\chi^{2}$-test with Fisher's correction performed on a Web-based statistical $\chi^{2}$ calculator (GraphPad.com). Results were deemed statistically significant if $P \leqslant 0.05$.

\section{RESULTS}

Characteristics of colonic neoplastic lesions. The characteristics (lesion size, colonic site of origin, adenomatous histology type and degree of dysplasia) of the 98 colorectal neoplastic lesions assessed for NGAL expression are presented in Table 1. Not all specimens were taken forward to analysis. On examination of the stained $\mathrm{CaP}$ sections, 1 was fragmented, 4 specimens contained carcinomatous epithelium in entirety with no adenomatous component and 14 did not contain carcinomatous epithelium. These were excluded from 


\begin{tabular}{|c|c|c|}
\hline Characteristics & $\begin{array}{l}\text { CaP identified } \\
\text { from diagnostic } \\
\text { database }(n=52)\end{array}$ & $\begin{array}{l}\text { Adenomas collected } \\
\text { within paired } \\
\text { polyp-normal } \\
\text { sample set }(n=46)\end{array}$ \\
\hline Colonic site & $\begin{array}{l}50(96 \%) \text { distal to } \\
\text { splenic flexure }\end{array}$ & $\begin{array}{l}38(83 \%) \text { distal to splenic } \\
\text { flexure }\end{array}$ \\
\hline Size & $\begin{array}{l}1(2 \%) \text { small }<1 \mathrm{~cm}, \\
51(98 \%) \text { large }>1 \mathrm{~cm}\end{array}$ & $\begin{array}{l}16(35 \%) \text { small }<1 \mathrm{~cm}, 30 \\
(65 \%) \text { large } \geqslant 1 \mathrm{~cm}\end{array}$ \\
\hline Size range & $0.8-3.1 \mathrm{~cm}$ & $0.3-3 \mathrm{~cm}$ \\
\hline $\begin{array}{l}\text { Histological } \\
\text { classification of } \\
\text { adenomatous } \\
\text { epithelium }\end{array}$ & $\begin{array}{l}31(\%) \text { tubular } \\
\text { adenoma, } 21 \text { (\%) } \\
\text { tubulo-villous } \\
\text { adenoma }^{a}\end{array}$ & $\begin{array}{l}41(89 \%) \text { tubular } \\
\text { adenoma, } 5(11 \%) \\
\text { tubulo-villous adenoma }\end{array}$ \\
\hline $\begin{array}{l}\text { Degree of dysplasia of } \\
\text { adenomatous } \\
\text { epithelium }\end{array}$ & $\begin{array}{l}4(7 \%) \text { LGD, } 12(27 \%) \\
\text { LGD/HGD, } 31(66 \%) \\
H_{G D}\end{array}$ & All LGD \\
\hline \multicolumn{3}{|c|}{$\begin{array}{l}\text { Abbreviations: } \mathrm{HGD}=\text { high-grade dysplasia; } L G D=\text { low-grade dysplasia; } L G D / H G D=L G D \\
\text { background with foci of } \mathrm{HGD} \text {. } \\
\mathrm{a}_{n=47 ;} \text { four } \mathrm{CaP} \text { lesions had no adenomatous epithelium seen on stained section and } \\
\text { therefore excluded. }\end{array}$} \\
\hline
\end{tabular}
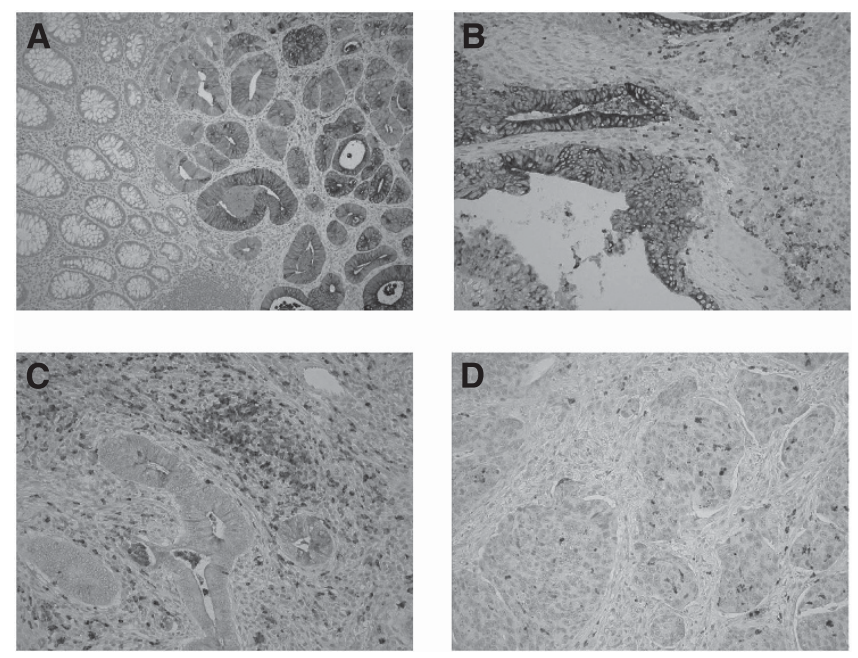

Figure 1. Expression of NGAL in colorectal neoplastic progression. (A) NGAL epithelial and stromal cell staining in the adenomatous compartment of a CaP lesion, with no positive staining seen within the normal mucosa ( $\times 20$ magnification). There is a clear demarcation in staining pattern between these compartments highlighting that NGAL expression occurs early in the adenoma-carcinoma sequence with a sharp transition between normal and dysplastic epithelium. (B) Epithelial and stromal cell NGAL positivity within colonic adenoma exhibiting low-grade dysplasia ( $\times 40$ magnification). (C, D) Epithelial and stromal cell NGAL positivity within carcinomatous epithelial compartment of a CaP lesion ( $\times 40$ magnification).

the analysis and therefore the final number of samples included in the analysis was 47 normal epithelial, 47 adenomatous epithelial and 33 carcinomatous epithelial compartments.

Expression of NGAL in CaP. There was a statistically significant increase in total NGAL expression in compartments displaying colorectal neoplasia compared with normal mucosa (Figure 1; Table 2). All (100\%) of the adenoma- and carcinomatous-defined compartments in $\mathrm{CaP}$ lesions showed NGAL immunopositivity
Table 2. Total NGAL expression in each histological compartment of $\mathrm{CaP}$ lesions (epithelial and stromal)

\begin{tabular}{|c|c|c|c|}
\hline & $\begin{array}{c}\text { Normal colonic } \\
\text { mucosa } \\
n=47^{\mathrm{a}, \mathrm{b}}\end{array}$ & $\begin{array}{c}\text { Adenoma } \\
n=47^{a, c}\end{array}$ & $\begin{array}{c}\text { Carcinoma } \\
n=33^{b, c}\end{array}$ \\
\hline NGAL positive & $2(4 \%)$ & 47 (100\%) & 33 (100\%) \\
\hline NGAL negative & 45 (96\%) & $0(0 \%)$ & $0(0 \%)$ \\
\hline \multicolumn{4}{|c|}{$\begin{array}{l}\text { Abbreviation: NGAL }=\text { neutrophil gelatinase-associated lipocalin. } \\
\text { a }_{\text {Normal vs }} \text { Adenoma, } \chi=82.5, P=0.0001 \\
\text { b }_{\text {Normal vs Carcinoma, }} \chi=68.4, P=0.0001 \\
{ }^{c} \text { Adenoma vs Carcinoma, not significant. }\end{array}$} \\
\hline
\end{tabular}

Table 3. Localisation of NGAL positivity (epithelial/stromal) in each histological compartment of CaP lesions

\begin{tabular}{|c|c|c|c|}
\hline & $\begin{array}{c}\text { Normal } \\
\text { colonic } \\
\text { mucosa } \\
n=47\left({ }^{*},{ }^{* \star}\right)\end{array}$ & $\begin{array}{c}\text { Adenoma } \\
n=47\left(^{\star},{ }^{\star \star \star}\right)\end{array}$ & $\begin{array}{c}\text { Carcinoma } \\
\left.n=33^{(\star \star, * \star *}\right)\end{array}$ \\
\hline Epithelial cell NGAL positive $^{a}$ & $0(0 \%)$ & $43(92 \%)$ & $19(58 \%)$ \\
\hline Epithelial cell NGAL negative $^{a}$ & 47 (100\%) & $4(8 \%)$ & $14(42 \%)$ \\
\hline Stromal cell positive ${ }^{\mathbf{b}}$ & $2(4 \%)$ & $46(98 \%)$ & $33(100 \%)$ \\
\hline Stomal cell negative ${ }^{\mathbf{b}}$ & 45 (96\%) & $1(2 \%)$ & $0(0 \%)$ \\
\hline \multicolumn{4}{|c|}{ 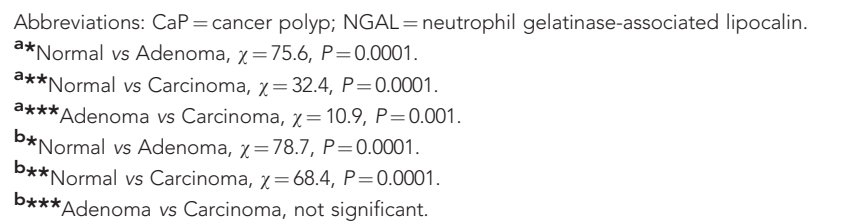 } \\
\hline
\end{tabular}

compared with only 4\% (2 out of 47) of normal mucosa $(P=0.0001)$. Considering the localisation of this immunoreactivity (Table 3), the two positive normal mucosal specimens exhibited stromal cell expression with no epithelial cell positivity identified in this tissue type. In contrast, 43 of the 47 (92\%) and 19 of the 33 (58\%) adenomatous and carcinomatous epithelium compartments, respectively, expressed NGAL, a significant increase compared with normal mucosa (normal vs adenoma, $\chi=75.6, P=0.0001$; normal $v s$ carcinoma, $\chi=32.4, P=0.0001)$. Notably, there was significantly less epithelial expression in carcinomatous epithelium compared with adenomatous epithelium in these $\mathrm{CaP}$ lesions $(\chi=10.9, P=0.001)$. Considering this epithelial expression, there was no difference in the intensity or proportion of positive epithelial cells seen between the adenomatous and carcinomatous epithelium (Figure 2). The majority of both the adenomatous and carcinomatous compartments expressed stromal cell positivity: 46 out of $47(98 \%)$ and 33 out of 33(100\%), respectively. As such, there was no statistical difference in stromal cell NGAL expression associated with neoplastic progression from adenoma to carcinoma $(\chi=0.711, \mathrm{NS})$. As only 4 out of 47 adenomatous compartments were negative for epithelial expression of NGAL, the association of this to differing histology type or degree of dysplasia was unable to be accessed in this sample group.

Expression of NGAL in normal mucosa-adenoma paired samples. There was a statistically significant increase in total NGAL expression in the adenomas compared with adjacent normal mucosa $(67 \%$ (31 out of 46$)$ vs $4 \%$ ( 1 out of 26 ), respectively, $\chi=26.7, P=0.0001)$. None of the normal mucosal 
In 1 (weak/moderate) $\quad \mathrm{N} 2$ (strong) $\quad \mathrm{P} 2(>50 \%)$

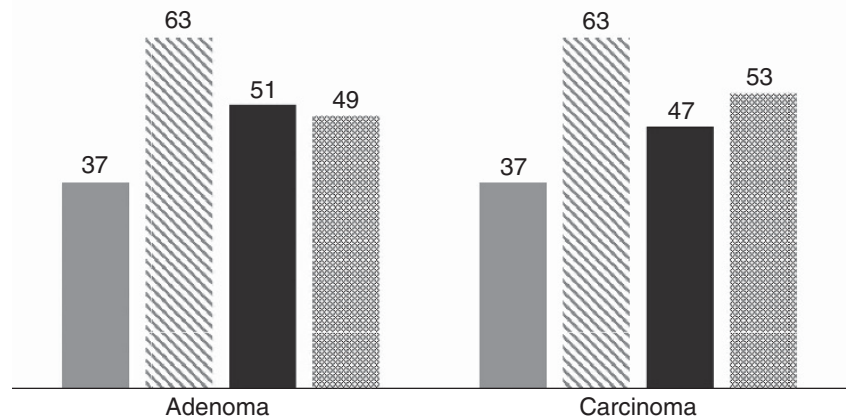

Figure 2. Proportion of positive epithelial cells and intensity of epithelial cell staining did not differ between adenomatous and carcinomatous epithelium within CaP lesions. Numerical values represent \% of total specimens/histological compartment. I= intensity; $\mathrm{P}=$ proportion of total tissue section.

\begin{tabular}{l} 
Table 4. Total NGAL expression related to adenoma size \\
\begin{tabular}{|l|c|c|}
\hline & $\begin{array}{c}\text { Small adenoma } \\
(<1 \mathrm{~cm}), \mathbf{n}=16\end{array}$ & $\begin{array}{r}\text { Large adenoma } \\
(\geqslant 1 \mathrm{~cm}), \mathbf{n}=\mathbf{3 0}\end{array}$ \\
\hline NGAL positive & 7 & 24 \\
\hline NGAL negative & 9 & 6 \\
\hline Abbreviation: NGAL = neutrophil gelatinase-associated lipocalin. $\chi=4.69, P=0.03$.
\end{tabular} \\
\hline
\end{tabular}

biopsies showed NGAL epithelial positivity. In comparison, almost half of the adenomatous lesions expressed epithelial NGAL (44\%, 20 out of 46), and this difference was statistically significant, $\lambda=13.56, P=0.0002$. This epithelial positivity was strong in intensity in 14 out of 20 adenomas, and in terms of proportion of positive staining, the majority exhibited positivity in less than half of the tissue area (13 out of 20). The one normal biopsy that was positive for NGAL staining showed stromal compartment immunoreactivity. In the adenoma group, 25 of the 46 (54\%) lesions showed NGAL stromal positivity (normal vs adenoma, $\lambda=16.24, P=0.0001)$.

NGAL expression in relation to adenoma size. Of the 46 adenoma lesions assessed, 30 were large at $\geqslant 1 \mathrm{~cm}$ in size and the remainder $(n=16)$ small at $<1 \mathrm{~cm}$ in size. Table 4 shows NGAL expression in relation to size. NGAL expression was greater in the larger adenoma group, and this reached statistical significance $(\chi=4.69, P=0.03)$.

\section{DISCUSSION}

In this paper, we report that NGAL is expressed by the majority of human neoplastic colorectal lesions, within both the epithelial and stromal cell compartments. This phenotypic switch occurs at a very early stage in neoplastic progression with a clear differential expression occurring between normal mucosa and adenomatous polyps, rather than further downstream in disease progression at the adenoma-carcinoma transformation. This was seen in two separate and differing cohorts of colorectal neoplastic tissue specimens representing each side of the neoplastic spectrum, independent on whether the adenomatous epithelium was within a low-risk low-grade dysplastic lesion or part of a polyp already harbouring a malignant focus. Not all adenomas proceed to malignancy, and it is estimated that $<5 \%$ of polyps will follow this fate. It is unclear why this is the case. Our aim was to investigate if expression of NGAL could be used as a protein biomarker of high-malignant potential and hence aid determination of colonoscopic surveillance interval in patients found to have adenomas on index colonoscopy. However, it is clear from our data that expression of NGAL per se cannot contribute to determining the malignant potential of such lesions and aid clinical decision making in this way.

Sun et al (2011) previously assessed NGAL expression in a large cohort of colorectal cancers and found a correlation with tumour progression and patient outcome in a Chinese population. In keeping with our data, NGAL was largely absent from normal colonic mucosa. In addition, they assessed expression in over 100 adenomas and reported epithelial positivity in $40 \%$ of their lowgrade adenoma cohort, and this increased to $85 \%$ within highgrade dysplastic epithelium. This is in keeping with our current data that shows epithelial NGAL expression in $92 \%$ of mainly high-grade dysplastic compartments of CaPs, and $67 \%$ of our lowrisk low-grade adenomas obtained from colonoscopy as part of the UK colorectal cancer screening program. In addition, we show that NGAL expression is linked to polyp size, another physical characteristic used to assess malignant potential.

It is not a surprise that stromal expression of NGAL is increased in adenomatous lesions. Neutrophils are the recognised primary inflammatory cell source of this protein, released into the surrounding environment as a defence mechanism as part of innate immunity. Our previous work revealed that the adenomatous microenvironment contains an increased immune cell infiltrate compared with the adjacent macroscopically normal mucosa (McLean et al, 2011), rich in neutrophil and macrophage cells and thus is in keeping with the current data. Similarly, Luley et al (2011) found increased levels of another granulocyte-derived protein calprotectin in adenomas compared with adjacent normal mucosa, and this correlated strongly with an increased neutrophil infiltrate seen in colonic polyps, comparable in magnitude to that in overt colorectal cancer. Similarly, myeloperoxidase positivity, used as an immunohistochemical marker of granulocyte infiltration, was increased in correlation with neoplastic progression, with increasing inflammatory cell infiltrates identified within aberrant crypt foci to adenomas to overt invasive malignancy (Roncucci et al, 2008).

The key questions revolve around the expression of NGAL in the epithelial compartment of the adenomatous lesions - what is its role in this setting, what triggers it's expression and what influence does it have on disease progression? Devireddy et al (2005) reported that iron-rich ligand binding to the $\mathrm{LCN}-2$ receptor increases intra-cellular iron and inhibits apoptosis, conferring a growth advantage. Jung et al (2012) showed macrophage-derived NGAL stimulates growth of in vitro breast cancer cell lines. In addition, expression signature of NGAL in breast cancer tissue follows disease progression, by expression of mesenchymal markers and loss of E-cadherin expression, promoting invasive disease (Yang et al, 2009). Indeed, in a colorectal cancer cell line, NGAL overexpression was linked to loss of cell-to-cell adhesion and increased invasion (Hu et al, 2009). Similarly, an in vivo mouse model of xenographic colon cancer linked increased expression of NGAL to increased tumour growth and metastatic disease (Sun et al, 2011). Another mechanism that may promote this phenotype is the formation of NGAL/MMP-9 complexes, protecting the latter proteolytic enzyme from degradation, with negative correlation to tissue degradation and invasion (Bolignano et al, 2010). However, Barresi et al (2011) reported expression of NGAL as a negative prognostic marker in early colorectal carcinoma that was not dependent on concurrent expression of MMP-9.

It is clear that NGAL can potentially promote tumorigenesis via several different mechanisms and this can differ in different tissue types (reviewed in Bolignano et al, 2010 and 
Chakraborty et al, 2012). This is supported by the finding that expression of NGAL during embryogenesis and development is tissue specific. In the gastrointestinal tract, NGAL is strongly expressed at an embryonic stage to 16 weeks of fetal development, and then vanishes (Zhang et al, 2012). Our data suggest that this expression then reappears in dysplastic colonic epithelia. NGAL has previously been reported in additional pre-malignant pathologies, such as endometrial (Miyamoto et al, 2011) and pancreatic (Moniaux et al, 2008) sites.

Overall, this study reveals that expression of NGAL in neoplastic colonic epithelium occurs very early in the adenoma-carcinoma sequence with the phenotypic switch seen at the normal to adenoma transition. Consequently, the majority of adenomas express NGAL, particularly with increasing adenoma size, and this may be important in the transition to malignancy. We aimed to assess whether NGAL could be used as a protein biomarker of malignant potential, and given the extensive expression rate in adenomas, this was not the case. However, the nature and the consequences of NGAL expression, particularly in the epithelial compartment, are of interest in understanding early colorectal carcinogenesis and warrant further investigation.

\section{REFERENCES}

Barresi V, Di Gregorio C, Reggiani-Bonetti L, Leni A, Ponz-De LM, Barresi G (2010) Neutrophil gelatinase-associated lipocalin: a new prognostic marker in stage I colorectal carcinoma? Human Pathol 42(11): 1720-1726.

Barresi V, Reggiani-Bonetti L, Di Gregorio C, Vitarelli E, Ponz De Leon M, Barresi G (2011) Neutrophil gelatinase-associated lipocalin (NGAL) and matrix metalloproteinase-9 (MMP-9) prognostic value in stage I colorectal carcinoma. Pathol Res Pract 207(8): 479-486.

Bolignano D, Donato V, Lacquaniti A, Fazio MR, Bono C, Coppolino G, Buemi M (2010) Neutrophil gelatinase-associated lipocalin (NGAL) in human neoplasias: a new protein enters the scene. Cancer Lett 208: 10-16.

Bratt T (2000) Lipocalins and cancer. Biochim Biophys Acta 1482(1-2): 318-326.

Cairns SR, Scholefield JH, Steele RJ, Dunlop MG, Thomas HJ, Evans GD, Eaden JA, Rutter MD, Atkin WP, Saunders BP, Lucassen A, Jenkins P, Fairclough PD, Woodhouse CR. British Society of Gastroenterology; Association of Coloproctology for Great Britain and Ireland (2010) Guidelines for colorectal cancer screening and surveillance in moderate and high risk groups (update from 2002). Gut 59: 666-689.

Chakraborty S, Kaur S, Guha S, Batra SK (2012) The multifaceted roles of neutrophil gelatinase associated lipocalin (NGAL) in inflammation and cancer. Biochim Biophys Acta 1826(1): 129-169.

Colucci PM, Yale SH, Rall CJ (2003) Colorectal polyps. Clin Med Res 1(3): 261-262.

Devireddy LR, Gazin C, Zhu X, Green MR (2005) A cell-surface receptor for lipocalin $24 \mathrm{p} 3$ selectively mediates apoptosis and iron uptake. Cell 23: 1293-1305.

Duncan R, Carpenter B, Main LC, Telfer C, Murray GI (2008) Characterisation and protein expression profiling of annexins in colorectal cancer. Br J Cancer 98: 426-433.

Fearon ER, Vogelstein B (1990) A genetic model for colorectal tumorigenesis. Cell 61(5): 759-767.

Giuliani A, Caporale A, Corona M, Ricciardulli T, Di Bari M, Demoro M, Scarpini M, Angelico F (2006) Large size, villous content and distal location are associated with severe dysplasia in colorectal adenomas. Anticancer Res 26(5B): 3717-3722.

Haggar FA, Boushey RP (2009) Colorectal cancer epidemiology: incidence, mortality, survival, and risk factors. Clin Colon Rectal Surg 22(4): 191-197.

Hu L, Hittelman W, Lu T, Ji P, Arlinghaus R, Shmulevich I, Hamilton SR, Zhang W (2009) NGAL decreases E-cadherin-mediated cell-cell adhesion and increases cell motility and invasion through Rac1 in colon carcinoma cells. Lab Invest 89(5): 531-548.

Jung M, Weigert A, Tausendschön M, Mora J, Oren B, Sola A, Hotter G, Muta T, Brüne B (2012) IL-10-induced NGAL production in macrophages with consequences for tumor growth. Mol Cell Biol 32(19): 3938-3948.

Leslie A, Carey FA, Pratt NR, Steele RJC (2002) The colorectal adenomacarcinoma sequence. Br J Surgery 89(7): 845-860.

Luley K, Noack F, Lehnert H, Homann N (2011) Local calprotectin production in colorectal cancer and polyps-active neutrophil recruitment in carcinogenesis. Int J Colorectal Dis 26(5): 603-607.

McLean MH, Murray GI, Fyfe M, Hold GL, Mowat NAG, El-Omar EM (2008) COX-2 expression in sporadic colorectal adenomatous polyps is linked to adenoma characteristics. Histopathology 52: 806-815.

McLean MH, Murray GI, Stewart KN, Norrie G, Mayer C, Hold GL, Thomson J, Fyfe N, Hope M, Mowat NA, Drew JE, El-Omar EM (2011) The inflammatory microenvironment in colorectal neoplasia. PLoS One 6(1): e15366.

Miyamoto T, Kashima H, Suzuki A, Kikuchi N, Konishi I, Seki N, Shiozawa T (2011) Laser-captured microdissection-microarray analysis of the genes involved in endometrial carcinogenesis: stepwise up-regulation of lipocalin2 expression in normal and neoplastic endometria and its functional relevance. Human Pathol 42: 1265-1274.

Moniaux N, Chakraborty S, Yalniz M, Gonzalez J, Shostrom VK, Standop J, Lele SM, Ouellette M, Pour PM, Sasson AR, Brand RE, Hollingsworth MA, Jain M, Batra SK (2008) Early diagnosis of pancreatic cancer: neutrophil gelatinase-associated lipocalin as a marker of pancreatic intraepithelial neoplasia. Br J Cancer 98(9): 1540-1547.

O’Brian MJ, Winawer SJ, Zauber AG, Gottlieb LS, Sternberg SS, Diaz B, Dickersin GR, Ewing S, Geller S, Kasimian D (1990) The National Polyp Study. Patient and polyp characteristics associated with high-grade dysplasia in colorectal adenomas. Gastoenterology 98(2): 371-379.

Roncucci L, Mora E, Mariani F, Bursi S, Pezzi A, Rossi G, Pedroni M, Luppi D, Santoro L, Monni S, Manenti A, Bertani A, Merighi A, Benatti P, Di Gregorio C, de Leon PM (2008) Myeloperoxidase-positive cell infiltration in colorectal carcinogenesis as indicator of colorectal cancer risk. Cancer Epidemiol Biomarkers Prev 17(9): 2291-2297.

Sun Y, Yokoi K, Li H, Gao J, Hu L, Liu B, Chen K, Hamilton SR, Fan D, Sun B, Zhang W (2011) NGAL expression is elevated in both colorectal adenoma- carcinoma sequence and cancer progression and enhances tumorigenesis in xenograft mouse models. Clin Cancer Res 17: 4331-4340.

Yang J, Bielenberg DR, Rodig SJ, Doiron R, Clifton MC, Kung AL, Strong RK, Zurakowski D, Moses MA (2009) Lipocalin 2 promotes breast cancer progression. Proc Natl Acad Sci USA 106(10): 3913-3918.

Zhang PX, Zhang FR, Xie JJ, Tao LH, Lü Z, Xu XE, Shen J, Xu LY, Li EM (2012) Expression of NGAL and NGALR in human embryonic, fetal and normal adult tissues. Mol Med Rep 6(4): 716-722.

This work is published under the standard license to publish agreement. After 12 months the work will become freely available and the license terms will switch to a Creative Commons AttributionNonCommercial-Share Alike 3.0 Unported License. 